\title{
Absolute Factoring of Non-holonomic Ideals in the Plane
}

\author{
D. Grigoriev \\ CNRS, Mathématiques, Université de Lille, 59655, Villeneuve d'Ascq, France, \\ e-mail: dmitry.grigoryev@math.univ-lille1.fr, \\ website: http://logic.pdmi.ras.ru/\%grigorev \\ F. Schwarz \\ FhG, Institut SCAI, 53754 Sankt Augustin, Germany, \\ e-mail: fritz.schwarz@scai.fraunhofer.de \\ website: www.scai.fraunhofer.de/schwarz.0.html
}

\begin{abstract}
We study non-holonomic overideals of a left differential ideal $J \subset F\left[\partial_{x}, \partial_{y}\right]$ in two variables where $F$ is a differentially closed field of characteristic zero. One can treat the problem of finding non-holonomic overideals as a generalization of the problem of factoring a linear partial differential operator. The main result states that a principal ideal $J=\langle P\rangle$ generated by an operator $P$ with a separable symbol $\operatorname{symb}(P)$ has a finite number of maximal non-holonomic overideals; the symbol is an algebraic polynomial in two variables. This statement is extended to non-holonomic ideals $J$ with a separable symbol. As an application we show that in case of a second-order operator $P$ the ideal $\langle P\rangle$ has an infinite number of maximal non-holonomic overideals iff $P$ is essentially ordinary. In case of a third-order operator $P$ we give sufficient conditions on $\langle P\rangle$ in order to have a finite number of maximal non-holonomic overideals. In the Appendix we study the problem of finding non-holonomic overideals of a principal ideal generated by a second order operator, the latter being equivalent to the Laplace problem. The possible application of some of these results for concrete factorization problems is pointed out.
\end{abstract}

AMS Subject Classifications: 35A25, 35C05, 35G05

Keywords: differential non-holonomic overideals, Newton polygon, formal series solutions.

\section{FINITENESS OF THE NUMBER OF MAX- IMAL NON-HOLONOMIC OVER-IDEALS OF AN IDEAL WITH SEPARABLE SYM- BOL}

Let $F$ be a differentially closed field (or universal differential field in terms of [8], [9]) with derivatives $\partial_{x}$ and $\partial_{y}$; let $P=\sum_{i, j} p_{i, j} \partial_{x}^{i} \partial_{y}^{j} \in F\left[\partial_{x}, \partial_{y}\right]$ be a partial differential operator of order $n$. Considering e.g. the field of rational

Permission to make digital or hard copies of all or part of this work for personal or classroom use is granted without fee provided that copies are not made or distributed for profit or commercial advantage and that copies bear this notice and the full citation on the first page. To copy otherwise, to republish, to post on servers or to redistribute to lists, requires prior specific permission and/or a fee.

ISSAC'10, July 20-23, 2010, München.

Copyright 2010 ACM 978-1-59593-904-3/08/07 ...\$10.00. functions $\mathbb{Q}(x, y)$ as $F$ is a quite different issue. The symbol is defined by $\operatorname{symb}(P)=\sum_{i+j=n} p_{i, j} v^{i} w^{j}$; it is a homogeneous algebraic polynomial of degree $n$ in two variables. The degree of its Hilbert-Kolchin polynomial $e z+e_{0}$ is called its differential type; its leading coefficient is called the typical differential dimension [8]. A left ideal $I \subset F\left[\partial_{x}, \partial_{y}\right]$ is called non-holonomic if its differential type equals 1 . We study maximal non-holonomic overideals of a principal ideal $\langle P\rangle \subset$ $F\left[\partial_{x}, \partial_{y}\right]$. Obviously there is an infinite number of maximal holonomic overideals of $\langle P\rangle$ : for any solution $u \in F$ of $P u=$ 0 we get a holonomic overideal $\left\langle\partial_{x}-u_{x} / u, \partial_{y}-u_{y} / u\right\rangle \supset\langle P\rangle$. We assume w.l.o.g. that $\operatorname{symb}(P)$ is not divisible by $\partial_{y}$; otherwise one can make a suitable transformation of the type $\partial_{x} \rightarrow \partial_{x}, \partial_{y} \rightarrow \partial_{y}+b \partial_{x}, b \in F$. In fact choosing $b$ from the subfield of constants of $F$ is possible.

Clearly, factoring an operator $P$ can be viewed as finding principal overideals of $\langle P\rangle$; we refer to factoring over a universal field $F$ as absolute factoring. Overideals of an ideal in connection with Loewy and primary decompositions were considered in [6].

Following [4] consider a homogeneous polynomial ideal $\operatorname{symb}(I) \subset F[v, w]$ and attach a homogeneous polynomial $g=G C D(\operatorname{symb}(I))$ to $I$. Lemma 4.1 [4] states that $\operatorname{deg}(g)=e$. As above one can assume w.l.o.g. that $w$ does not divide $g$.

We recall that the Ore ring $R=\left(F\left[\partial_{y}\right]\right)^{-1} F\left[\partial_{x}, \partial_{y}\right]$ (see [1]) consists of fractions of the form $\beta^{-1} r$ where $\beta \in F\left[\partial_{y}\right]$, $r \in F\left[\partial_{x}, \partial_{y}\right]$, see [3], [4]. We also recall that one can represent $R=F\left[\partial_{x}, \partial_{y}\right]\left(F\left[\partial_{y}\right]\right)^{-1}$, and two fractions are equal, $\beta^{-1} r=r_{1} \beta_{1}^{-1}$, iff $\beta r_{1}=r \beta_{1}$ [3], [4].

For a non-holonomic ideal $I$ denote ideal $\bar{I}=R I \subset R$. Since the ring $R$ is left-euclidean (as well as right-euclidean) with respect to $\partial_{x}$ over the skew-field $\left(F\left[\partial_{y}\right]\right)^{-1} F\left[\partial_{y}\right]$, we conclude that the ideal $\bar{I}$ is principal. Let $\bar{I}=\langle r\rangle$ for suitable $r \in F\left[\partial_{x}, \partial_{y}\right] \subset R$ (cf. [4]). Lemma 4.3 [4] implies that $\operatorname{symb}(r)=w^{m} g$ for a certain integer $m \geq 0$ where $g$ is not divisible by $w$.

Now we expose a construction introduced in [4]. For a family of elements $f_{1}, \ldots, f_{k} \in F$ and rational numbers $s_{i} \in \mathbb{Q}, 1>s_{2}>\cdots>s_{k}>0$ we consider a $D$-module being a vector space over $F$ with a basis $\left\{G^{(s)}\right\}_{s \in \mathbb{Q}}$ where the derivatives of

$$
G^{(s)}=G^{(s)}\left(f_{1}, \ldots, f_{k} ; s_{2}, \ldots, s_{k}\right)
$$


are defined as

$d_{x_{i}} G^{(s)}=\left(d_{x_{i}} f_{1}\right) G^{(s+1)}+\left(d_{x_{i}} f_{2}\right) G^{\left(s+s_{2}\right)}+\cdots+\left(d_{x_{i}} f_{k}\right) G^{\left(s+s_{k}\right)}$

for $i=1,2$ using the notations $d_{x_{1}}=\partial_{x}, d_{x_{2}}=\partial_{y}$.

Next we introduce series of the form

$$
\sum_{0 \leq i<\infty} h_{i} G^{\left(s-\frac{i}{q}\right)}
$$

where $q$ is the least common multiple of the denominators of $s_{2}, \ldots, s_{k}$; one can view (1) as an analogue of NewtonPuiseux series for non-holonomic $D$-modules. Theorem 2.5 [4] states that for any linear divisor $v+a w$ of $\operatorname{symb}(P)$ and any $f_{1} \in F$ such that $\left(\partial_{x}+a \partial_{y}\right) f_{1}=0$ there exists a solution of $P=0$ of the form (1); conversely, if (1) is a solution of $P=$ 0 then $\left(\partial_{x}+a \partial_{y}\right) f_{1}=0$ for an appropriate divisor $v+a w$ of $\operatorname{symb}(P)$. Furthermore, Proposition 4.4 [4] implies that any solution of theform (1) of $r=0$ such that $\left(\partial_{x}+a \partial_{y}\right) f_{1}=0$ for suitable $a \in F$ (or equivalently $\partial_{y} f_{1} \neq 0$ ) is also a solution of the ideal $I$; then the appropriate linear form $v+a w$ is a divisor of $g$, and the inverse holds as well.

In [5] we have designed an algorithm for factoring an operator $P$ in case of $\operatorname{symb}(P)$ is separable. In particular, in this case there is only a finite number (less than $2^{n}$ ) of different factorizations of $P$. Now we show a more general statement for overideals of $\langle P\rangle$.

TheOREM 1.1. Let $\operatorname{symb}(P)$ be separable. Then there exists at most $n=\operatorname{ord}(P)$ maximal non-holonomic overideals of $\langle P\rangle \subset F\left[\partial_{x}, \partial_{y}\right]$. Moreover, if there exists a nonholonomic overideal $I \supset\langle P\rangle$ with the attached polynomial $g=G C D(\operatorname{symb}(I))$ then there exists a unique non-holonomic overideal, maximal among the ones with the attached polynomial equal $\mathrm{g}$.

Proof. Let $I$ be a non-holonomic ideal such that $I \supset\langle P\rangle$. Then $\beta P=r_{1} r$ for suitable $\beta \in F\left[\partial_{y}\right], r_{1} \in$ $F\left[\partial_{x}, \partial_{y}\right]$ and a polynomial $g=G C D(\operatorname{symb}(I))$ attached to $I$ is a divisor of $\operatorname{symb}(P)$. We claim that for every pair of non-holonomic ideals $I_{1}, I_{2} \supset\langle P\rangle$ to which a fixed polynomial $g$ is attached, to their sum $I_{1}+I_{2}$ also $g$ is attached. Indeed, any solution of the form (1) of $P=0$ such that $(v+a w) \mid g$, is a solution of $r=0$ as well due to Lemma 4.2 [4] (cf. Proposition 4.4 [4]) taking into account that symb(P) is separable, hence it is also a solution of $I$ as it was shown above and by the same token is a solution of both $I_{1}$ and $I_{2}$ (in particular $I_{1}+I_{2}$ is also non-holonomic). The claim is established.

Thus among non-holonomic overideals $I \supset\langle P\rangle$ to which a given polynomial $g \mid \operatorname{sym} b(P)$ is attached, there is a unique maximal one. Now take two maximal non-holonomic overideals $I, I^{\prime} \supset\langle P\rangle$ to which polynomials $g, g^{\prime}$ are attached, respectively. Then $g, g^{\prime}$ are reciprocately prime. Indeed, if $v+a w$ divides both $g, g^{\prime}$ then arguing as above one can verify that (1) is a solution of $I+I^{\prime}$, i.e. the latter ideal is non-holonomic which contradicts to maximality of $I, I^{\prime}$. Theorem is proved.

Corollary 1.2. Let symb $(P)$ be separable. Suppose that there exist maximal non-holonomic overideals $I_{1}, \ldots, I_{l} \supset$ $\langle P\rangle$ such that for the respective attached polynomials $g_{1}, \ldots, g_{l}$ the sum of their degrees $\operatorname{deg}\left(g_{1}\right)+\cdots+\operatorname{deg}\left(g_{l}\right) \geq n$. The $\langle P\rangle=$ $I_{1} \cap \cdots \cap I_{l}$.

Proof. As it was shown in the proof of Theorem 1.1, polynomials $g_{j} \mid \operatorname{symb}(P), 1 \leq j \leq l$ are pairwise reciprocately prime, hence $g_{1} \cdots g_{l}=\operatorname{symb}(P)$. Moreover it was established in the proof of Theorem 1.1 that every solution of $P=0$ of the form (1) such that $\left(\partial_{x}+a \partial_{y}\right) f_{1}=0$, is a solution of a unique $I_{j}$ for which $(u+a w) \mid g_{j}$; thus every solution of $P=0$ of the form (1) is also a solution of $I_{1} \cap \cdots \cap I_{l}$. Therefore the typical differential dimension of ideal the $I_{1} \cap \cdots \cap I_{l}$ equals $n$ (cf. Lemma 4.1 [4]). On the other hand, any overideal of a principal ideal $\langle P\rangle$ of the same typical differential dimension coincides with $\langle P\rangle$; one can verify it by comparing their Janet bases [10]. (We briefly recall that operators $P_{1}, \ldots, P_{s} \in F\left[\partial_{x}, \partial_{y}\right]$ form a Janet basis of the ideal $\left\langle P_{1}, \ldots, P_{s}\right\rangle$ if for any element $P \in\left\langle P_{1}, \ldots, P_{s}\right\rangle$ its highest derivative $l d(P)$ is divided by one of $l d\left(P_{i}\right), 1 \leq i \leq s$.)

REMARK 1.3. One can extend Theorem 1.1 to non-holonomic ideals $J$ such that the homogeneous polynomial $G C D(\operatorname{symb}(J))$ is separable: namely, there exists a finite number of maximal non-holonomic overideals $I \supset J$.

\section{NON-HOLONOMIC OVERIDEALS OF A SECOND-ORDER LINEAR PARTIAL DIF- FERENTIAL OPERATOR}

In this Section we study the structure of overideals of $\langle P\rangle$ when $n=\operatorname{ord}(P)=2$. The case of separable $\operatorname{symb}(P)$ is covered by Theorem 1.1.

Proposition 2.1. Any principal ideal $\langle P\rangle$ for a secondorder operator $P=\partial_{y}^{2}+p_{1} \partial_{x}+p_{2} \partial_{y}+p_{3}$ with non-separable $\operatorname{symb}(P)$ has

i) no proper non-holonomic overideals in case $p_{1} \neq 0$;

ii) an infinite number of maximal non-holonomic overideals in case $p_{1}=0$.

Proof. Let $\operatorname{sym} b(P)$ be non-separable. Then applying a transformation of the type $\partial_{x} \rightarrow b_{1} \partial_{x}+b_{2} \partial_{y}, \partial_{y} \rightarrow$ $b_{3} \partial_{x}+b_{4} \partial_{y}$ for suitable $b_{1}, b_{2}, b_{3}, b_{4} \in F$ one can assume w.l.o.g. that $P=\partial_{y}^{2}+p_{1} \partial_{x}+p_{2} \partial_{y}+p_{3}$; it would be interesting to find out when one can carry out these transformations algorithmically. First let $p_{1}=0$. Then $P$ is essentially ordinary, i.e. becomes ordinary after a transformation as above, and for any solution $u \in F$ of the equation $P=0$ we get a non-holomonic overideal $\left\langle\partial_{y}-u_{y} / u\right\rangle \supset\langle P\rangle$. Now suppose that $p_{1} \neq 0$. Then $P$ is irreducible (see e. g. Corollary $7.1[4])$. Moreover we claim that $\langle P\rangle$ has at most one maximal non-holonomic overideal. Let $I \supset\langle P\rangle$ be a nonholonomic overideal. Choosing arbitrary non-zero elements $b_{1}, b_{2} \in F$ denote the derivation $d=b_{1} \partial_{x}+b_{2} \partial_{y}$. Similar to the proof of Theorem 1.1 there exists $r \in F\left[d, \partial_{y}\right]=$ $F\left[\partial_{x}, \partial_{y}\right]$ such that $\langle r\rangle=I R_{1} \subset R_{1}=(F[d])^{-1} F\left[d, \partial_{y}\right]$. Then $\beta P=r_{1} r$ for suitable $\beta \in F[d], r_{1} \in F\left[d, \partial_{y}\right]$ and $\operatorname{symb}(r)=\left(b_{1} v+b_{2} w\right)^{m} g$ for an integer $m$ and $g \mid w^{2}$. If $g=1$ then $I$ cannot be non-holonomic because of Proposition 4.4 [4] (cf. above). If $g=w^{2}$ then similar to the proof of Corollary 1.2 one can show that the only non-holonomic overideal of $\langle P\rangle$ among ones to which polynomial $w^{2}$ is attached, is just $\langle P\rangle$ itself. It remains to consider the case $g=w$. Applying the Newton polygon construction from [4] to equation $r=0$ and a divisor $w$ of $\operatorname{symb}(r)$, one obtains a solution of the form (1) of $r=0$ with $G=G(x)$, thereby it is a solution of $P=0$. On the other hand, applying the Newton polygon construction from [4] to equation $P=0$, one gets at its first step $f_{1}=x$ and at the second step $f_{2}$ which fulfils equation $\left(\partial_{y} f_{2}\right)^{2}+p_{1}=0$ and $f_{2}$ corresponds to the edge of the Newton polygon with endpoints $(0,2)$ and $(1,0)$, 
so with the slope $1 / 2$. This provides a solution of equation $P=0$ of the form (1) with $G=G\left(x, f_{2} ; 1 / 2\right)$, therefore the equation $P=0$ has no solutions of the form (1) with $G=G(x)$. The achieved contradiction shows that there are no non-holonomic overideals $I$ with attached polynomial $w$, this completes the proof of the claim.

\section{ON NON-HOLONOMIC OVERIDEALS OF A THIRD-ORDER OPERATOR}

Now we study overideals of $\langle P\rangle$ where the order $n=$ $\operatorname{ord}(P)=3$. Due to Theorem 1.1 it remains to consider nonseparable $\operatorname{symb}(P)$. In [4] an algorithm has been designed for factoring $P$; a few explicit calculations for factoring $P$ are provided in [7].

Proposition 3.1. Let $P$ be a third-order operator with a non-separable symb $(P)$.

i) When symb $(P)$ has two different linear divisors, one of which of multiplicity 2, then we can assume w.l.o.g. that

$$
P=\partial_{x} \partial_{y}^{2}+p_{0} \partial_{x}^{2}+p_{1} \partial_{x} \partial_{y}+p_{2} \partial_{y}^{2}+p_{3} \partial_{x}+p_{4} \partial_{y}+p_{5} .
$$

If $p_{0} \neq 0$ then $\langle P\rangle$ has at most two maximal non-holonomic overideals. Moreover if there exist two different maximal non-holonomic overideals $I_{1}, I_{2} \supset\langle P\rangle$ then $\langle P\rangle=I_{1} \cap I_{2}$;

ii) When $\operatorname{symb}(P)$ has a single linear divisor of multiplicity 3 we can assume w.l.o.g. that

$$
P=\partial_{y}^{3}+p_{0} \partial_{x}^{2}+p_{1} \partial_{x} \partial_{y}+p_{2} \partial_{y}^{2}+p_{3} \partial_{x}+p_{4} \partial_{y}+p_{5} .
$$

If either $p_{0} \neq 0$, either $p_{2} \neq 0$ or $p_{3} \neq 0$ then $\langle P\rangle$ has at most two maximal non-holonomic overideals. Moreover if there exist two different maximal non-holonomic overideals $I_{1}, I_{2} \supset\langle P\rangle$ then $\langle P\rangle=I_{1} \cap I_{2}$. Otherwise $\left\langle P=\partial_{y}^{3}+p_{2} \partial_{y}^{2}+\right.$ $\left.p_{4} \partial_{y}+p_{5}\right\rangle$ has an infinite number of maximal non-holonomic overideals.

Proof. Case $i)$ First let $\operatorname{symb}(P)$ have two linear divisors; therefore one can assume w.l.o.g. (see above) that $w$ is its divisor of multiplicity 2 and $v$ is its divisor of multiplicity 1. One can write

$$
P=\partial_{x} \partial_{y}^{2}+p_{0} \partial_{x}^{2}+p_{1} \partial_{x} \partial_{y}+p_{2} \partial_{y}^{2}+p_{3} \partial_{x}+p_{4} \partial_{y}+p_{5} .
$$

Suppose that $p_{0} \neq 0$. The Newton polygon construction from [4] applied to equation $P=0$ and to divisor $w$ of $\operatorname{symb}(P)$, yields a solution of the form (1) of $P=0$ with $f_{1}=x$ at its first step. At its second step the construction yields $f_{2}$ which fulfils equation $\left(\partial_{y} f_{2}\right)^{2}+p_{0}=0$ and which corresponds to the edge of the Newton polygon with endpoints $(1,2),(2,0)$, so with the slope $1 / 2$. This provides $G=G\left(x, f_{2} ; 1 / 2\right)$ in (1).

Let a non-holonomic ideal $I \supset\langle P\rangle$. Choose $d=b_{1} \partial_{x}+$ $b_{2} \partial_{y}$ for non-zero $b_{1}, b_{2} \in F$. As in the previous Section there exists $r \in F\left[d, \partial_{y}\right]$ such that $\langle r\rangle=R_{1} I \subset R_{1}=$ $(F[d])^{-1} F\left[d, \partial_{y}\right]$. Then $\beta P=r_{1} r$ for suitable $\beta \in F[d], r_{1} \in$ $F\left[d, \partial_{y}\right]$. Rewrite $\operatorname{symb}(r)=\left(b_{1} v+b_{2} w\right)^{m} g$ where $g \mid\left(v w^{2}\right)$. If either $g=w^{2}$ or $g=v$, one can argue as in the proof of Theorem 1.1 and deduce that there can exist at most one maximal non-holonomic overideal of $\langle P\rangle$ with the property that the polynomial attached to the overideal is either $w^{2}$ or $v$. Similar to the proof of Corollary 1.2 one can verify that if there exist maximal non-holonomic overideals $I_{2}, I_{1} \supset\langle P\rangle$ with attached polynomials $w^{2}$ and $v$, then $\langle P\rangle=I_{1} \cap I_{2}$. As in Theorem 1.1 the existence of a maximal overideal with the attached polynomial $w^{2}$ or $v$ follows from the existence of any non-holonomic overideal with the attached polynomial $w^{2}$ or $v$.

If either $g=w$ or $g=v w$ then applying the Newton polygon construction from [4] to equation $r=0$ and divisor $w$ of $\operatorname{symb}(r)$, one obtains a solution of $r=0$ (and thereby, of $P=0$ due to Lemma 4.2 [4]) of the form (1) with $G=G(x)$ which contradicts to the supposition $p_{0} \neq 0$ (see above). Thus, in case $p_{0} \neq 0$ the ideal $\langle P\rangle$ has a finite number, less or equal than 2 , of maximal non-holonomic overideals (similar to Theorem 1.1).

When $p_{0}=0$ this is not always true, say for $P=\left(\partial_{x}+\right.$ b) $\left(\partial_{y}^{2}+b_{3} \partial_{y}+b_{4}\right)$ (cf. case $n=2$ in the previous Section). It would be interesting to clarify for which $P$ this is still true.

Case $i$ i) Now we consider the last case when $\operatorname{symb}(P)$ has a unique linear divisor with multiplicity 3 . As above one can assume w.l.o.g. that $\operatorname{symb}(P)=w^{3}$, so

$$
P=\partial_{y}^{3}+p_{0} \partial_{x}^{2}+p_{1} \partial_{x} \partial_{y}+p_{2} \partial_{y}^{2}+p_{3} \partial_{x}+p_{4} \partial_{y}+p_{5} .
$$

Keeping the notations we get $\langle r\rangle=R_{1} I$ and $\beta P=r_{1} r$. Then $\operatorname{symb}(r)=\left(b_{1} v+b_{2} w\right)^{m} g$ where $g \mid w^{3}$. If $g=w^{3}$ then arguing as in the proof of Corollary 1.2 we deduce that the only non-holonomic overideal of $\langle P\rangle$ to which polynomial $w^{3}$ is attached, is just $\langle P\rangle$ itself.

Let $g \mid w^{2}$. Applying the Newton polygon construction from [4] to equation $r=0$ and linear divisor $w$ of $\operatorname{symb}(r)$ one gets a solution of $r=0$ (and thereby of $P=0$ ) with either $G=G(x)$ or $G=G\left(x, f_{2} ; 1 / 2\right)$ where $\partial_{y} f_{2} \neq 0$ (cf. above).

Application of the Newton polygon construction from [4] to equation $P=0$ (and unique linear divisor $w$ of $\operatorname{symb}(P)$ ) at its first step provides $f_{1}=x$. The second step requires a trial of cases. First let $p_{0} \neq 0$. Then the second step yields $f_{2}$ which fulfils equation $\left(\partial_{y} f_{2}\right)^{3}+p_{0}=0$ and which corresponds to the edge of the Newton polygon with endpoints $(0,3),(2.0)$, so with the slope $2 / 3$. Thus we obtain a solution of the form (1) with $G=G\left(x, f_{2}, \ldots ; 2 / 3, \ldots\right)$, hence $\langle P\rangle$ in case $p_{0} \neq 0$ has no non-holonomic overideals with attached polynomial $g$ being a divisor of $w^{2}$ (see above).

Now assume that $p_{0}=0$ and $p_{1} \neq 0$. Then the second step provides solutions of $P=0$ of the form (1) with two different possibilities. Either the Newton polygon construction chooses the vertical edge with endpoints $(1,1),(1,0)$ as a leading edge at the second step, then it terminates at the second step yielding a solution of the form (1) with $G=G(x)$; we recall that in the construction from Section 2 [4] only edges with non-negative slopes are taken as leading ones and the construction terminates while taking a vertical edge, so with the slope 0 , as a leading one, in particular the edge with endpoints $(1,1),(1,0)$ is taken as a leading one regardless of whether the coefficient at point $(1,0)$ vanishes. As the second possibility the construction yields a solution of the form (1) with $G=G\left(x, f_{2}, \ldots ; 1 / 2, \ldots\right)$ where $f_{2} \neq 0$ fulfils equation $\left(\partial_{y} f_{2}\right)^{3}+p_{1} \partial_{y} f_{2}=0$ corresponding to the edge of the Newton polygon with endpoints $(0,3),(1,1)$, so with the slope $1 / 2$. One can suppose w.l.o.g. that the Newton polygon construction terminates at its third step (thereby $G=G\left(x, f_{2} ; 1 / 2\right)$ ), otherwise $\langle P\rangle$ cannot have a non-holonomic overideal to which a divisor $g$ of $w^{2}$ is attached (see above).

If $g=w^{2}$ then any solution $H_{2}$ of $P=0$ of the form (1) with $G=G\left(x, f_{2} ; 1 / 2\right)$ is a solution of $r=0$ because otherwise $r \mathrm{H}_{2} \neq 0$, being also of the form (1) with $G=G\left(x, f_{2} ; 1 / 2\right)$, cannot be a solution of $r_{1}=0$ tak- 
ing into account that $\operatorname{symb}\left(r_{1}\right)$ does not divide on $w^{2}$ (cf. Lemma 4.2 [4]). Else if $g=w$ then $r H_{2} \neq 0$ (again taking into account that $\operatorname{symb}(r)$ does not divide on $\left.w^{2}\right)$ and therefore $r_{1}\left(r H_{2}\right)=0$. Hence for a solution $H_{1}$ of $P=0$ of the form (1) with $G=G(x)$ (see above) we have $r H_{1}=0$ since otherwise $r H_{1}$ being also of the form (1) with $G=G(x)$ cannot be a solution of $r_{1}=0$ (again cf. Lemma 4.2 [4]). Then arguing as in the proof of Theorem 1.1 one concludes that in case $p_{0}=0$ and $p_{1} \neq 0$ ideal $\langle P\rangle$ can have at most two maximal non-holonomic overideals with attached polynomials $w$ and $w^{2}$. Similar to the proof of Corollary 1.2 (cf. the preceding Subsection) one can verify that if there exist maximal non-holonomic overideals $I_{1}, I_{2} \supset\langle P\rangle$ with attached polynomials $w$ and $w^{2}$, then $\langle P\rangle=I_{1} \cap I_{2}$. As in Theorem 1.1 the existence of a maximal overideal with the attached polynomial $w$ (or respectively, $w^{2}$ ) follows from the existence of any non-holonomic overideal with the attached polynomial $w$ or $w^{2}$.

Furthermore, let $p_{0}=p_{1}=0, p_{3} \neq 0$. Then as in case $p_{0} \neq 0$ we argue that the second step of the Newton polygon construction applied to equation $P=0$ yields $f_{2}$ which fulfils equation $\left(\partial_{y} f_{2}\right)^{3}+p_{4}=0$ and which corresponds to the leading edge of the Newton polygon with endpoints $(0,3),(1,0)$, so with the slope $1 / 3$. Thus the Newton polygon construction yields a solution of $P=0$ of the form (1) with $G=G\left(x, f_{2}, \ldots ; 1 / 3, \ldots\right)$ and again $\langle P\rangle$ in case $p_{0}=p_{1}=0, p_{3} \neq 0$ under consideration has no nonholonomic overideals with an attached polynomial being a divisor of $w^{2}$.

Finally, when $p_{0}=p_{1}=p_{3}=0$ the ideal $\left\langle P=\partial_{y}^{3}+\right.$ $\left.p_{1} \partial_{y}^{2}+p_{3} \partial_{y}+p_{5}\right\rangle$ has an infinite number of maximal nonholonomic overideals; this is similar to the second-order case $P=\partial_{y}^{2}+p_{4} \partial_{y}+p_{5}$, see above.

A few examples applying the preceding result are given next.

EXAMPLE 1. The operator

$$
L \equiv \partial_{y y}+x \partial_{x}+\partial_{y}+y
$$

is immediately recognized as absolutely irreducible by case $i$ ) of Proposition 2.1 because $p_{1} \neq 0$.

ExAmple 2. Consider the operator

$$
L \equiv \partial_{x y y}+\partial_{x x}+y \partial_{y y}+(y+1) \partial_{x}+2 \partial_{y}+y .
$$

Due to $p_{0}=1$, case $i$ ) of the above proposition applies. In fact, there is only a single first-order right factor as may be seen from

$$
L=\left(\partial_{y y}+\partial_{x}+1\right)\left(\partial_{x}+y\right)
$$

this decomposition may be obtained by using the function FirstOrderRightFactors provided on the website www .alltypes.de [11].

Example 3. Case ii) of Proposition 3.1 applies to the operator

$L \equiv \partial_{y y y}+\frac{x}{y^{2}} \partial_{x y}+\left(1+\frac{2}{y}\right) \partial_{y y}+\frac{x(y-2)}{y^{3}} \partial_{x}+\frac{2 y-3}{y^{2}} \partial_{y}-\frac{y-2}{y^{3}} ;$

although $p_{0}=0$, due to $p_{2} \neq 0$ and $p_{3} \neq 0$ the operator can have at most two different right factors. It turns out that there are no first-order right factors at all.
It is a challenge to design an algorithm which produces non-holonomic overideals of a given differential ideal $J \subset$ $F\left[\partial_{x}, \partial_{y}\right]$ in general. If the goal is solving linear pde's attached to these operators, $F=\mathbb{Q}(x, y)$ is of particular interest. Some of the results reported in this article may be applied for obtaining a partial answer; e.g. by case $i$ ) of Proposition 2.1 it may be possible to exclude the existence of any factor very efficiently.

\section{Appendix. Explicit formulas for Laplace trans- formation}

We exhibit a short exposition and explicit formulas for the Laplace transformation [2]. Let $Q=\partial_{x y}+a \partial_{x}+b \partial_{y}+c$ be a second-order operator which has its Laplace divisor $L_{n}=$ $\sum_{0<i<n} l_{i} \partial_{x}^{i}$ of order $n$, i. e. $Q, L_{n}$ form a Janet basis of ideal $\left\langle Q, L_{n}\right\rangle$. Hence

$$
P Q=\left(\partial_{y}+a\right) L_{n}
$$

for a suitable $P=\sum_{0<i<n-1} p_{i} \partial_{x}^{i}$. (This form of $P$ is obtained by comparing the highest terms which divide on $\partial_{x}^{n}$ in (2).)

If a Laplace divisor exists then $\left\langle Q, L_{n}\right\rangle$ is a proper nonholonomic overideal of $\langle Q\rangle$. Conversely, one can show (cf. $[2])$ that if $\langle Q\rangle$ has a proper non-holonomic overideal then there exists either a Laplace divisor $L_{n}$ (for a suitable $n$ ) or a Laplace divisor of the form $\sum_{0 \leq i<n} t_{i} \partial_{y}^{i}$ with respect to $\partial_{y}$. That is why the problem of searching for a Laplace divisor is equivalent to finding non-holonomic proper overideals of $\langle Q\rangle$.

Open question: is there an algorithm which decides for a given $Q$ whether it has a Laplace divisor? In particular, an upper bound on $n$ would suffice for an algorithm.

Comparing the highest terms in (2) which divide on $\partial_{y}$, we get that $L_{n}=P\left(\partial_{x}+b\right)$. Thus

$$
P Q=\left(\partial_{y}+a\right) P\left(\partial_{x}+b\right) .
$$

We have $Q \neq\left(\partial_{y}+a\right)\left(\partial_{x}+b\right)$ iff $0 \neq a b+b_{y}-c \equiv K_{0}$.

Lemma 3.2. If $K_{0} \neq 0$ then there are unique $B, C$ such that

$$
\left(\partial_{x}+B\right) Q=\left(d_{x y}+a \partial_{x}+B \partial_{y}+C\right)\left(\partial_{x}+b\right)
$$

Proof. (4) is equivalent to an algebraic linear system in $B, C$,

$$
\begin{array}{r}
a B-C=b_{y}+a b-a_{x}-c, \\
\left(c-b_{y}\right) B-b C=b_{x y}+a b_{x}-c_{x}
\end{array}
$$

Therefore (3) holds iff $P=P_{1}\left(\partial_{x}+B\right)$ by means of dividing $P$ by $\partial_{x}+B$ with remainder. Substituting the latter equality to (3) and making use of (4) we obtain the equality

$$
P_{1}\left(\partial_{x y}+a \partial_{x}+B \partial_{y}+C\right)=\left(\partial_{y}+a\right) P_{1}\left(\partial_{x}+B\right) .
$$

Now (7) is similar to (3) but with the order $\operatorname{ord}\left(P_{1}\right)=$ $\operatorname{ord}(P)-1=n-1$ and a new second-order operator $Q_{1}=\partial_{x y}+a \partial_{x}+B \partial_{y}+C$. Continuing this way we get the Laplace transformation with $K_{1}=a B+B_{y}-C$ etc.

More uniformly denote $b_{0} \equiv b, c_{0} \equiv c$, then $b_{1} \equiv B$, $c_{1} \equiv C, b_{2}, c_{2}$ etc. obtained from Lemma 3.2. Denote

$$
K_{i} \equiv a b_{i}+\left(b_{i}\right)_{y}-c_{i}, Q_{i} \equiv \partial_{x y}+a \partial_{x}+b_{i} \partial_{y}+c_{i} .
$$


COROLLARY 3.3. There exists $L_{n}$ satisfying (2) iff for the minimal $m$ such that $K_{m}=0$ we have $m \leq n$. In this case

$$
L_{n}=P_{n-m}\left(\partial_{x}+b_{m-1}\right) \cdots\left(\partial_{x}+b_{0}\right)
$$

where $P_{n-m}=\sum_{0<i<n-m} p_{i} \partial_{x}^{i}$ is an arbitrary operator of the order $n-m$ which fulfils

$$
P_{n-m}\left(\partial_{y}+a\right)=\left(\partial_{y}+a\right) P_{n-m} .
$$

For any order $n-m \geq 0$ such an operator $P_{n-m}$ exists. The pair $Q, L_{n}$ constitutes a Janet basis of the ideal $\left\langle Q, L_{n}\right\rangle$. The ideal $\left\langle Q, L_{m}\right\rangle$ is the unique maximal non-holonomic overideal of $\langle Q\rangle$ which corresponds to a divisor y of $\operatorname{symb}(Q)=$ xy (see Theorem 1.1).

Proof. Applying Laplace transformations as above, if $m>n$ we don't get a solution of (2) after $n$ steps since (3) with $P Q_{n}=\left(\partial_{y}+a\right) P\left(\partial_{x}+b_{n}\right)$ would not have a solution with $P$ of the order 0 . If $m \leq n$ then successively following Laplace transformations we arrive to (8) in which (9) is obtained from equality $P Q_{m}=\left(\partial_{y}+a\right) P\left(\partial_{x}+b_{m}\right)$ (see (3)) and taking into account that $K_{m}=0$.

ACKNOWLEDGEMENT The first author is grateful to the Max-Planck Institut für Mathematik, Bonn for its hospitality while writing this paper.

\section{REFERENCES}

[1] J. E. Björk, Rings of differential operators, North-Holland, 1979.

[2] E. Goursat, Leçon sur l'intégration des équations aux dérivées partielles, vol. I, II, A. Hermann, 1898.

[3] D. Grigoriev, Weak Bézout Inequality for D-Modules, J. Complexity 21 (2005), 532-542.

[4] D. Grigoriev, Analogue of Newton-Puiseux series for non-holonomic D-modules and factoring, Moscow Math. J. 9 (2009), 775-800.

[5] D. Grigoriev, F. Schwarz, Factoring and solving linear partial differential equations, Computing $\mathbf{7 3}$ (2004), 179-197.

[6] D. Grigoriev, F. Schwarz, Loewy and primary decomposition of D-Modules, Adv. Appl. Math. 38 (2007), 526-541.

[7] D. Grigoriev, F. Schwarz, Loewy decomposition of linear third-order PDE's in the plane, Proc. Intern. Symp. Symbolic, Algebr. Comput., ACM Press, $277-286$.

[8] E. Kolchin, Differential Algebra and Algebraic Groups, Academic Press, New York, 1973.

[9] M. van der Put, M. Singer, Galois theory of linear differential equations, Grundlehren der Mathematischen Wissenschaften, 328, Springer, 2003.

[10] F. Schwarz, Janet bases for symmetry groups, Groebner bases and applications, in London Math. Society, Lecture Note Ser. 251, 221-234, Cambridge University Press, Cambridge, 1998.

[11] F. Schwarz, ALLTYPES in the Web, ACM Communications in Computer Algebra, Vol. 42, No. 3, page 185-187(2008). 